\title{
The Impact of the Early Stages of Radio Source Evolution on the ISM of the Host Galaxies
}

\author{
R. Morganti ${ }^{1}$, C. N. Tadhunter ${ }^{2}$, T. A. Oosterloo ${ }^{1}$, \\ J. Holt ${ }^{2}$, A. Tzioumis ${ }^{3}$ and K. Wills ${ }^{2}$ \\ ${ }^{1}$ ASTRON, PO Box 2, 7990 AA Dwingeloo, The Netherlands \\ morganti@astron.nl \\ ${ }^{2}$ University of Sheffield, Hicks Building, Hounsfield Road, Sheffield, S3 7RH, UK \\ c.tadhunter@sheffield.ac.uk \\ j.holt@ sheffield.ac.uk \\ k.wills@sheffield.ac.uk \\ ${ }^{3}$ ATNF, PO Box 76, Epping NSW 1710, Australia \\ atzioumi@atnf.csiro.au
}

Received 2002 July 07, accepted 2002 December 13

\begin{abstract}
The study of both neutral and ionised gas in young radio sources is providing key information on the effect the radio plasma has on the ISM of these objects. We present results obtained for the compact radio sources PKS 1549-79, 4C 12.50 and PKS 1814-63 and for the intermediate-size radio galaxy 3C 459. At least in the first two, low ionisation optical emission lines and Hi absorption appear to be associated with the extended, but relatively quiescent, dusty cocoon surrounding the nucleus. The [OIII] lines are, on the other hand, mostly associated with the region of interaction between the radio plasma and the ISM, indicating a fast outflow from the centre. A case of fast outflow (up to $\sim 1000 \mathrm{~km} \mathrm{~s}^{-1}$ ) is also observed in $\mathrm{HI}$ in the radio source $4 \mathrm{C} 12.50$. As the radio source evolves, any obscuring material along the radio axis is swept aside until, eventually, cavities (of the same kind as observed e.g. in Cygnus A) are hollowed out on either side of the nucleus. We may witness this phase in the evolution of a radio source in the radio galaxy $3 \mathrm{C} 459$.
\end{abstract}

Keywords: galaxies: ISM — galaxies: active — radio lines: galaxies

\section{Introduction}

Although in the recent years important progress has been made in the understanding of the physics of AGN, a number of main questions still remain open. One of them is the early evolution of powerful radio galaxies. To know more about this phase is important not only from the point of view of the detailed phenomenology of these objects, but also for understanding the evolution of massive galaxies. In general, galaxies in their early stage of radio activity are likely to have their nuclear regions enshrouded in a cocoon of material left over from the accretion event(s) that trigger the activity. As the activity evolves, and the radio jets expand, the ISM and dust along the radio axis will be swept from the nuclear regions by jet-cloud interactions and/or quasar-induced winds. Thus, outflows of gas can be significant in this phase. These processes have been suggested to profoundly affect even the star formation history in luminous galaxies (Silk \& Rees 1998). Thus, the study of the early phase of radio activity and its effect on the galaxy medium has broad implications.

As described in many contributions in these proceedings, both neutral and ionised gas are clearly present around young radio sources. The study of this gas has a key role in the investigation of the evolutionary scenario described above. Spectral line observations can, in fact, provide information on the gas kinematics where signatures of interaction with the radio plasma can be found. Here we present the results (some still preliminary) obtained in the study of the $\mathrm{HI}$ in three compact radio sources (PKS 1549-79, 4C12.50 and PKS 1814-63) and one intermediate-size radio galaxy (3C 459$)$. While the HI detected in compact radio sources is very often interpreted as due to a circum-nuclear disk/torus, it is now clear that more complicated situations are present (see e.g. Morganti 2002, and references therein) and that neutral gas can also trace the presence of (sometimes extreme) interactions. The objects described here are also part of a detailed study of their ionised gas. This information is crucial for the correct interpretation of the kinematics of the neutral hydrogen, as will be illustrated below.

\section{The Case of PKS 1549-79}

In the above context, we have recently studied the southern radio galaxy PKS 1549-79. This is a compact radio source with a one-sided jet on the VLBI scale (see Figure 1). The overall size is about $350 \mathrm{pc}^{1}{ }^{1}$ The radio structure suggests that the radio jet axis is aligned close to our line of sight (King 1996). Because of these characteristics, it was quite surprising to detect $\mathrm{HI}$ absorption in this object (Morganti et al. 2001). The absorption has a peak optical depth of $\sim 2 \%$ (see Figure 1) and a column density of $\sim 4 \times 10^{20} T_{\text {spin }} / 100 \mathrm{~K} \mathrm{~cm}^{-2}$.

The optical spectrum of PKS 1549-79 is rich in strong emission lines (Tadhunter et al. 2001). Interestingly, the

\footnotetext{
${ }^{1}$ We use here $H_{0}=50 \mathrm{~km} \mathrm{~s}^{-1} \mathrm{Mpc}^{-1}$ and $q_{0}=0$.
} 


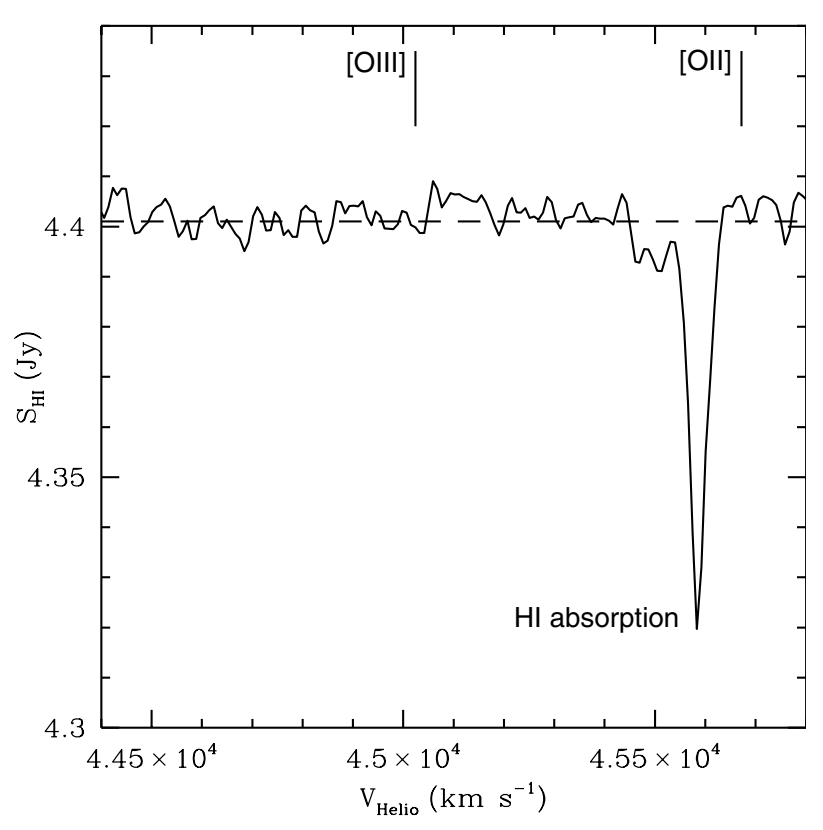

Figure 1 Left: Profile of the Hi absorption in PKS 1549-79 (from Morganti et al. 2001). The velocities derived from the optical emission lines are indicated. Right: Continuum radio emission on the VLBI scale from King (1996).

high ionisation ([OIII], [NeIII] and [Nev]) lines are unusually broad (FWHM $\sim 1200 \mathrm{~km} \mathrm{~s}^{-1}$ ) and blueshifted by $600 \mathrm{~km} \mathrm{~s}^{-1}$ relative to the much narrower low ionisation (i.e. [OII] and [OI]) lines. The remarkable result is that the velocity of the $\mathrm{HI}$ absorption agrees quite well with that derived from the low ionisation optical lines (see Figure 1).

Our interpretation of all this (Tadhunter et al. 2001) is that the low ionisation emission lines and Hi absorption are associated with the extended, but relatively quiescent, dusty cocoon surrounding the nucleus, whereas the [OIII] lines are associated with the region of interaction between the radio plasma and the ISM and indicating a fast outflow from the centre. We cannot yet fully exclude the possibility of starburst or AGN winds as responsible for the interaction. However, the common presence of broad (forbidden) lines in young, powerful radio sources (Gelderman \& Wittle 1994) seems to point to the radio jet as the likely candidate to produce the interaction. A cartoon of the proposed model is presented in Figure 3 in Holt, Tadhunter, \& Morganti (2003) and described in more details in Tadhunter et al. (2001). The interaction of the radio plasma with this gas would explain also the larger FWHM of the high ionisation lines.

\section{The Gas Outflow in $4 \mathrm{C} 12.50$}

One other object known to have optical characteristics similar to PKS 1549-79, i.e. two redshift systems and very broad optical lines, is the gigahertz peaked galaxy 4C 12.50 (see Grandi 1977). New optical spectra obtained for this object are described in detail in Holt et al. (2003). Three Gaussian components are needed to fit the strong [OIII] lines. One of them is a low ionisation component

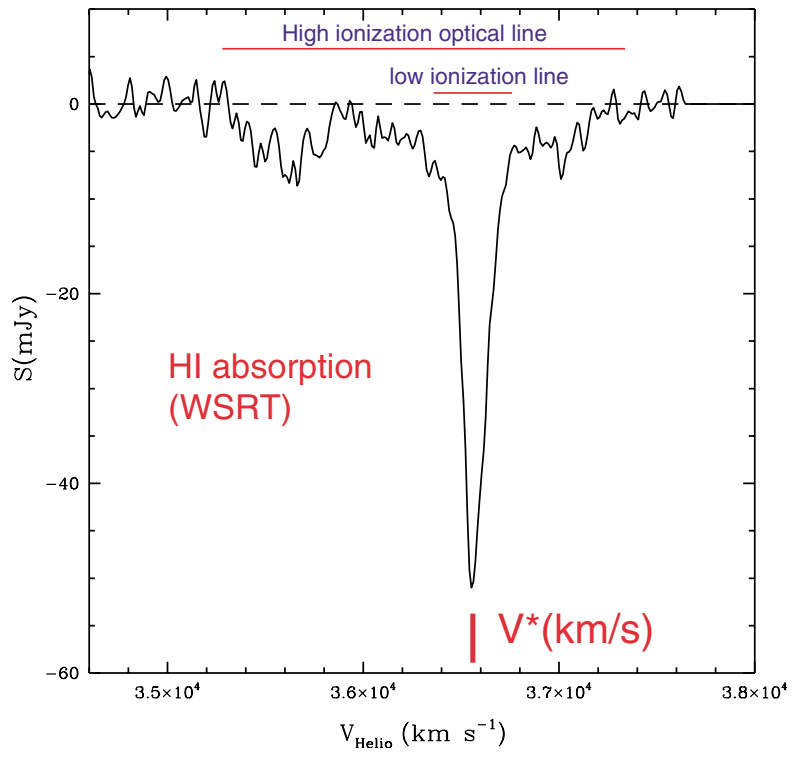

Figure 2 Profile of the $\mathrm{HI}$ absorption in 4C 12.50 as obtained from WSRT observations. The systemic velocity from stellar absorption lines (Grandi 1977) is marked. The range in velocity of two of the optical emission line components is also indicated (see Holt et al. 2003 for details).

and it is centred on the systemic velocity (which is derived by Grandi (1977) from the stellar absorption lines). The other two components show much higher ionisation and are highly blueshifted. The situation appears, therefore, similar to PKS 1549-79.

The presence of Hi absorption in this galaxy was already known from Arecibo observations (Mirabel 1989). However, new WSRT observations show that this absorption is actually much broader than previously known. As shown in Figure 2, an extremely broad absorption system is detected together with a (relatively) narrow and deeper component. This (relatively) narrow absorption $(\tau \sim 0.01$, column density $\sim 2 \times 10^{20} T_{\text {spin }} / 100 \mathrm{~K} \mathrm{~cm}^{-2}$ ) has a velocity similar to the systemic velocity detected from the stellar component and similar to the low ionisation component. Also in HI the situation appears, therefore, similar to PKS 1549-79. On the other hand, the overall system of shallower $\mathrm{HI}$ absorption $(\tau \sim 0.002$, column density $\sim 10^{20} T_{\text {spin }} / 100 \mathrm{~K} \mathrm{~cm}^{-2}$ ) is about $2000 \mathrm{~km} \mathrm{~s}^{-1}$ broad with a very large blueshifted wing (more than $1000 \mathrm{~km} \mathrm{~s}^{-1}$ ) as well as a redshifted wing of few hundred $\mathrm{km} \mathrm{s}^{-1}$. This broad absorption may represent an outflow of gas due to the interaction of the radio plasma with the dense ISM (e.g. gas in a cocoon around or entrained by the radio jet?). The blueshifted component would then be associated to the jet pointing toward us (and the opposite for the redshifted component). VLBI observations have now been obtained for $4 \mathrm{C} 12.50$ to investigate the detailed morphology of the $\mathrm{HI}$ absorption on the pc scale. The range of velocity of the broad $\mathrm{HI}$ absorption appears consistent with one of the high ionisation components found in the emission lines (see Holt et al. 2003). In this galaxy, therefore, the gas outflow would be seen both in the ionised and in the neutral 


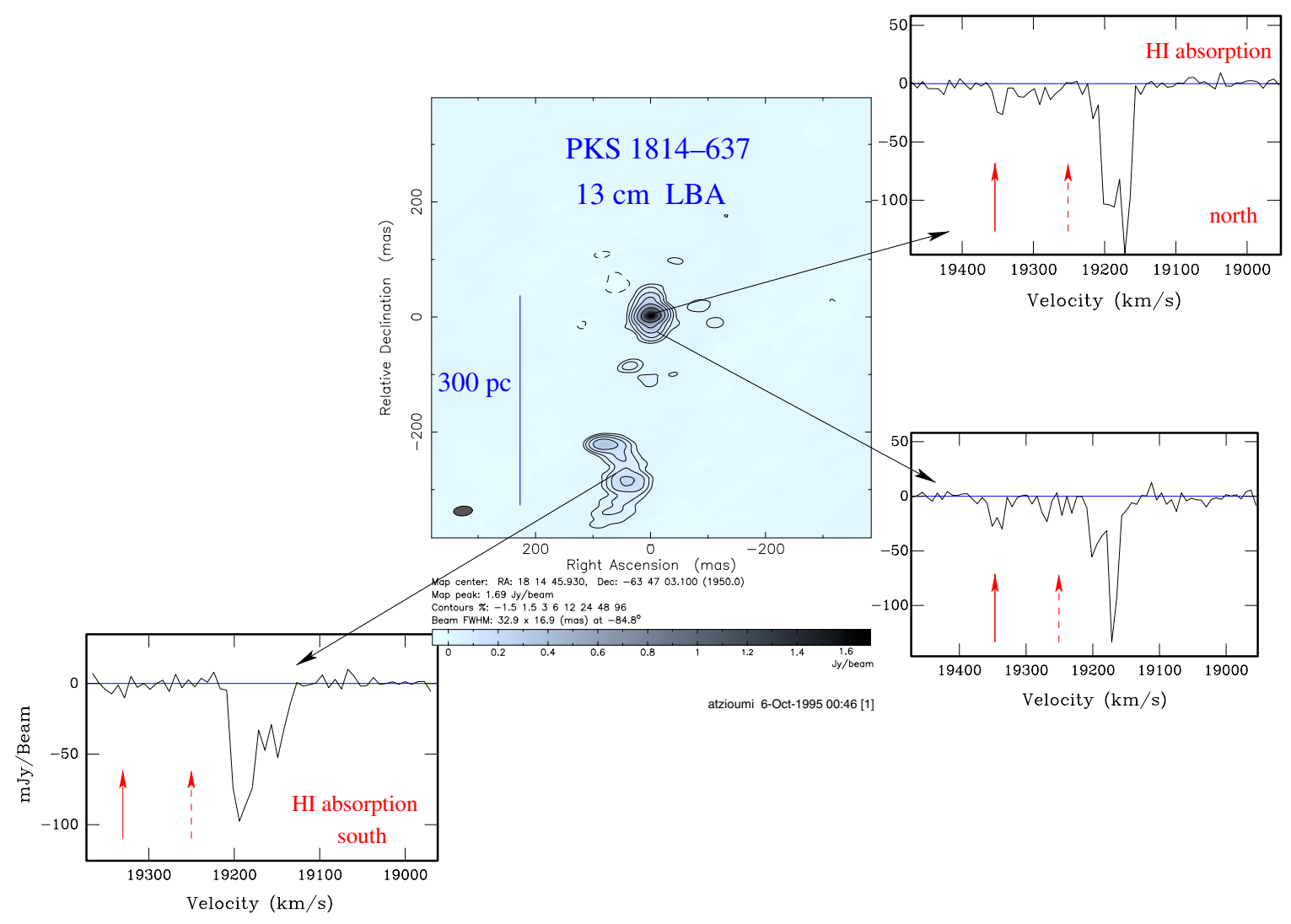

Figure 3 Continuum image of PKS 1814-63 at $13 \mathrm{~cm}$ and spectra of the Hi absorption obtained at three different locations in the source. The vertical lines indicate different values quoted for the systemic velocity of this galaxy.

gas. The scenario presented for PKS 1549-79 appears to explain also what is observed in $4 \mathrm{C} 12.50$ with the radio plasma being in the process of clearing its way through the rich ISM. It is however not yet clear how gas associated with such a fast outflow can remain neutral and not fully ionised. A fast outflow seen in neutral hydrogen is, however, not unique to $4 \mathrm{C} 12.50$. We have at least one other case in the radio-loud Seyfert 2 galaxy IC 5063 (Oosterloo et al. 2000). In this case, the radio plasma ejected from the nucleus is believed to interact directly with a molecular cloud.

\section{A Promising Case: PKS 1814-63?}

Another promising case is the compact (steep spectrum) radio galaxy PKS 1814-63. Hi absorption was detected from low-resolution observations (Morganti et al. 2001) and then followed up by LBA observations. Thus, unlike the sources described above, for PKS 1814-63 the distribution of the HI on the VLBI scale is known. On this scale, we detect $\mathrm{HI}$ absorption against the entire radio source (about $350 \mathrm{pc}$, see Figure 3). Unfortunately, the optical redshift is still quite uncertain (two of the values found in the literature are indicated in Figure 3) due to the poor quality of the available optical spectra. However, new high-quality optical spectra have been obtained very recently (but not yet fully analysed) and they show complex kinematics of the ionised gas. Until more information from the optical spectra is obtained, the interpretation of the kinematics of the neutral gas is still ambiguous.

The deep absorption (which has a very high value of optical depth $\tau \sim 0.2$, Morganti et al. 2001) could be due to a large-scale gas system. The fact that this absorption seems to have more components and their relative ratio changes for different positions against the radio source, could be connected with the presence of sub-structures in the gas screen. However, if the redshift of $19350 \mathrm{~km} \mathrm{~s}^{-1}$ is confirmed (drawn line in Figure 3, see Morganti et al. 2001 for a discussion), this gas could also be undergoing a mild outflow perhaps connected to the expansion of the source in the surrounding medium. If this is the case, we expect to see kinematical signatures also in the optical spectrum (like in PKS 1549-79 or 4C 12.50). Finding the actual systemic velocity will also help us in interpreting the shallow, broader component. This is detected only against the northern part of the radio source and could be due to a circumnuclear disk structure, although it is not clear yet from the available radio data whether this component actually corresponds to the core.

Finally, it is clear that in the study of PKS 1814-63 we are strongly limited in the interpretation of the HI absorption by the lack of more detailed optical information.

\section{The Intermediate Scale: $3 C 459$}

The above examples indicate that, at least in some cases, the obscuring material may cover a large fraction of the sky 


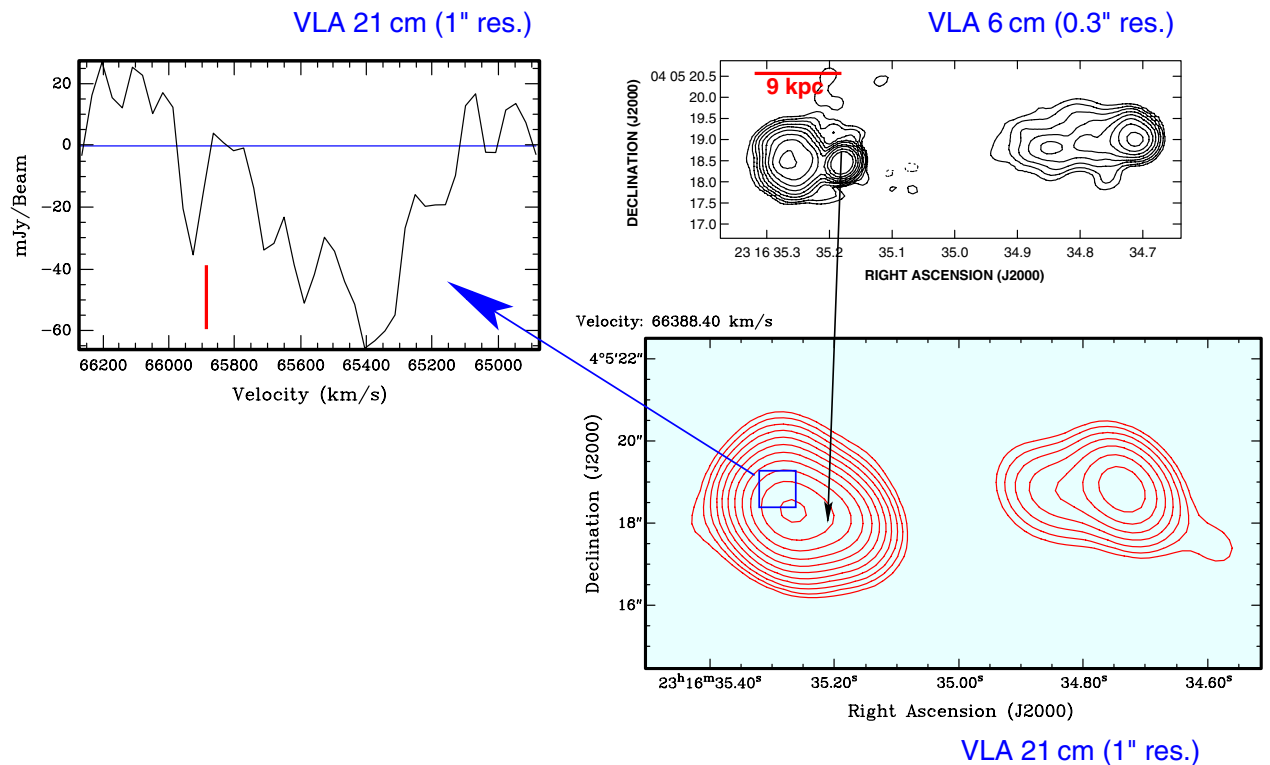

Figure 4 Radio continuum image of 3C 459 from VLA $6 \mathrm{~cm}$ (top panel) from Morganti et al. (1999) and from the line-free channels of the VLA $21 \mathrm{~cm}$ (lower panel). Left panel: Hi absorption spectra. The systemic velocity (from Spinrad et al. 1985) is indicated.

as seen by the central source. As the radio source evolves, any obscuring material along the radio axis is likely to be swept aside and dissipated by jet-cloud interactions or quasar-induced winds until, eventually, cavities are hollowed out on either side of the nucleus. Such cavities have been detected, for example, in the powerful extended radio source Cygnus A (Tadhunter et al. 1999). Before this stage is reached a substantial amount of material may be present along the radio axis, but at larger distances from the nucleus.

An intermediate case that we are studying in some detail is $3 \mathrm{C} 459$. This is a small and very asymmetric radio source. Its total size $(\sim 40 \mathrm{kpc})$ is much larger than the typical compact steep spectrum (CSS) source. Hi absorption has been detected in this source (Morganti et al. 2001) and new higher resolution observations (A-array VLA) tentatively show that most of the absorption could be located against the eastern radio lobe (see Figure 4) at about $9 \mathrm{kpc}$ from the radio core. Thus, this could be a case of an intermediate-size object where we can study the evolution of the gas after the most compact phase of the CSS source is past but when the source still has a galactic size. Furthermore, there could also be a relationship between the small size of the eastern component and the presence of a large amount of gas on that side of the source.

It is important to point out that the two compact objects mentioned above (PKS 1549-79 and 4C 12.50), as well as 3C 459, are far-IR bright galaxies and show, in their optical spectra, the presence of a young stellar population (we do not yet have information about PKS 1814-63). This is a particularly interesting class of objects where HI absorption is often detected. This could therefore point to the possibility that these objects are actually 'special' because of their particularly rich ISM.

\section{Summary}

We have shown that (at least some) compact radio galaxies can have different gas components with very different kinematics. The neutral hydrogen does not appear to be associated only with circumnuclear disks/tori (as often claimed), but instead there is evidence of the presence of more diffuse and quiescent halos in which these young sources are embedded. The radio jets are expected to clear their way through the rich ISM. As an indication of this, high speed outflows are detected not only in the ionised gas but, quite surprisingly, also in neutral hydrogen in the case of 4C 12.50 .

The kinematics of the neutral hydrogen alone can sometimes be difficult to unambiguously interpret. However, it can provide crucial information on the condition, morphology and kinematics of the gas in the central regions of radio sources once it is combined with the results from the ionised gas. This has been clearly shown for the case of the third radio galaxy presented, PKS 1814-63. Despite the complex and intriguing HI absorption detected, the interpretation is still uncertain due to the lack of high quality optical spectra. Finally, we have presented a case of an intermediate-size radio source (3C 459) where we may witness the next step in the evolution of the source.

In how many young radio sources do we expect to see the effect of the radio plasma making its way through the ISM and producing gas outflows? We do not yet have enough information available, especially in the optical (e.g. accurate measurement of the redshift), to estimate this in a reliable way. The group of radio galaxies that are far-IR bright and that show a young stellar population in their optical spectra seems, however, to be the most likely to show these extreme effects and outflows. 


\section{References}

Gelderman, R., \& Whittle, M. 1994, ApJS, 91, 491 Grandi, S. A. 1977, ApJ, 215, 446

Holt, J., Tadhunter, C. N., \& Morganti, R. 2003, PASA, 20, 25

King, E. 1996, PhD Thesis, University of Tasmania

Mirabel, I. F. 1989, ApJ, 340, L13

Morganti, R. 2002, in Issues in Unification of AGN, ASP Conf. Series 258, ed. R. Maiolino, A. Marconi, \& N. Nagar (San Francisco: ASP), 63 (astro-ph/0109056)

Morganti, R., Oosterloo, T. A., Tadhunter, C. N., Aiudi, R., Jones, P., \& Villar-Martin, M. 1999, A\&AS, 140, 355
Morganti, R., Oosterloo, T., Tadhunter, C. N., van Moorsel, G., Killeen, N., \& Wills, K. A. 2001, MNRAS, 323, 331

Oosterloo, T. A., Morganti, R., Tzioumis, A., Reynolds, J., King, E., McCulloch, P., \& Tsvetanov, Z. 2000, AJ, 119, 2085

Silk, J., \& Rees, M. J. 1998, A\&A, 331, L1

Spinrad, H., Djorgovski, S., Marr, J., \& Aguilar, L. 1985, PASA, 97, 932

Tadhunter, C. N., Wills, K. A., Morganti, R., Oosterloo, T., \& Dickson, R. 2001, MNRAS, 327, 227

Tadhunter, C. N., et al. 1999, ApJL, 512, L91 